\title{
The impact of the parabolic dish concentrator on the wind induced heat loss from its receiver
}

\author{
M. Uzair ${ }^{1,2}$, T.N. Anderson ${ }^{1}$, and R.J. Nates ${ }^{1}$ \\ ${ }^{1}$ School of Engineering, Auckland University of Technology, Auckland, New Zealand \\ ${ }^{2}$ NED University of Engineering \& Technology, Karachi, Pakistan \\ E-mail: muhammad.uzair@aut.ac.nz
}

\begin{abstract}
To achieve higher operating temperatures, power output and system efficiencies in parabolic dish cavity receivers, larger dish sizes and structures are used to increase the concentration ratio. This increases capital investment and installation costs, which in turn places a much stronger emphasis on accurately predicting the performance of the system and the heat loss from it. Numerous studies have investigated the natural convection heat losses from cavity receivers, and some have examined a cavity exposed to wind. However, the effect of the dish on the wind flow and subsequently the heat loss from the receiver has not been widely considered.

In this work, computational fluid dynamics was used to model the flow of air around a parabolic dish concentrator operating at varying angles of operation. The flow fields were validated using wind tunnel testing and published data regarding the aerodynamic characteristics of parabolic dishes. The results showed that the orientation of the dish has a significant effect on the flow structure near the receiver. Subsequently, using the validated method, the convective heat loss from the receiver of a large parabolic dish system was determined for a range of operating conditions.
\end{abstract}

The results support the assertion that the flow characteristics near the cavity receiver aperture depend strongly on the orientation of the dish structure. This resulted in the calculated heat loss being up to $40 \%$ lower than previous studies where the presence of the dish was included. As such, the wind flow around the dish needs to be accounted for when analyzing the performance of parabolic dish systems to avoid an overly conservative and hence more expensive design.

Keywords: parabolic dish; heat loss; wind; CSP

\section{Introduction}

Concentrating solar power (CSP) systems can be classified into four main types: parabolic dish systems, solar towers, parabolic troughs and Fresnel reflectors. Among these classifications, parabolic dish systems are considered to be the most efficient as a result of them achieving higher concentration ratios than the other techniques (Steinfield, 2005 and Tyner et al., 2001). To compete with conventional power generation techniques, the thermal performance of these CSP systems plays an important role. The performance of a CSP system utilizing a parabolic dish is sensitive to heat losses from the cavity receivers employed in these systems, particularly at high temperature. In particular, the heat loss from the cavity receiver is affected by the surrounding air motion, and consequently exposure to this could result in increased heat loss and decreased thermal performance (Lupfert et al., 2001).

Analytical techniques are available to determine the radiation and conduction heat losses from cavity receiver; however due to the complicated velocity and temperature field around the receiver, determination of convective heat losses is less straightforward. In this regard a significant body of research has developed with a view to understanding the convective heat 
losss from the cavity receiver of parabolic dish systems (Wu et al, 2010), particularly under natural convection conditions. In two of the most frequently cited works, Clausing (1981) and Clausing et al. (1987) investigated the natural convection heat losses from large cavities and proposed an analytical solution. In their implicit solution, the cavity receiver was divided into a convective zone and a stagnant zone. Similarly, Le Quere et al. (1981a,b) performed a numerical and experimental study in order to develop a relationship between the Nusselt number and tilt angle. In this vein, numerous further studies (Siebers and Kraabel,1984; Stine and McDonald, 1989; Leibfried and Ortjohann, 1995) have attempted to quantify and deliver a relationship between the natural convection heat transfer from parabolic dish receivers and their orientation (tilt angle), aperture size and cavity geometry.

Now in reality parabolic dish receivers are likely to be exposed to some degree of forced convection, however investigations of forced covection heat loss from these receivers are relatively scarce compared to those focussing on natural convection. In his study Ma (1993) examined a parabolic dish receiver and came to the conclusion that convection losses due to wind varied strongly with the receiver's tilt angle. More recently, Paitoonsurikarn and Lovegrove (2006) and Paitoonsurikarn et al. (2004) numerically examined how variations in wind velocity near a cavity receiver influenced the heat loss from it and developed a relationship describing this.

Now in almost all studies, researchers have treated the cavity receiver as an isolated entity, decoupled from the dish/reflector structure of a real parabolic dish system. Wu et al (2010) highlighted this issue in their review of the field, noting that there is a dearth of information relating to wind effects on heat loss and the interaction between the wind and dish, and by extension the influence on the heat loss. In this regard, the present study set out to address some of the issues highlighted by $\mathrm{Wu}$ et al (2010) by examining the heat loss from a parabolic dish cavity receiver due to the wind flow around the parabolic dish.

\section{Method}

\subsection{Numerical Setup}

In order to examine the effect of wind flow on the heat loss from parabolic dish receivers it was decided to undertake a computational fluid dynamics (CFD) analysis of the flow around the dish and receiver at varying angles of attack. For this study, the geometry chosen was that of the Australian National University's $20 \mathrm{~m}^{2}$ dish and frustum-shaped receiver described by Paitoonsurikarn and Lovegrove (2003). This parabolic dish has a focal length of $1.84 \mathrm{~m}$ and an aperture diameter of $5 \mathrm{~m}$ with a rim angle of approximately $70^{\circ}$; the receiver has dimensions as shown in Fig 1.

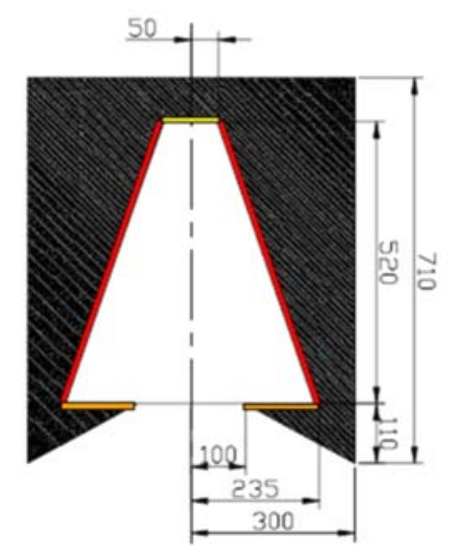

Fig. 1: Frustum shaped receiver and dimensions 
In undertaking the analysis, the computational domain around the dish-receiver system was extended $75 \mathrm{~m}$ upstream of the dish, $105 \mathrm{~m}$ downstream and $30 \mathrm{~m}$ in the lateral direction (Fig 2) to ensure all flow features were adequately captured, and walls were modelled using a no-slip boundary condition. A mesh sensitivity analysis was undertaken to ensure that the grid size did not influence the heat loss from the receiver. This resulted in a highly refined mesh of approximately 2 million elements being used to perform a steady state simulation of the flow around the dish. In constructing the mesh, finer grid elements were used near the receiver and in the wake region of the dish in order to predict the flow behaviour accurately. Regions further from the dish were meshed with larger grid sizes to improve computational speed.

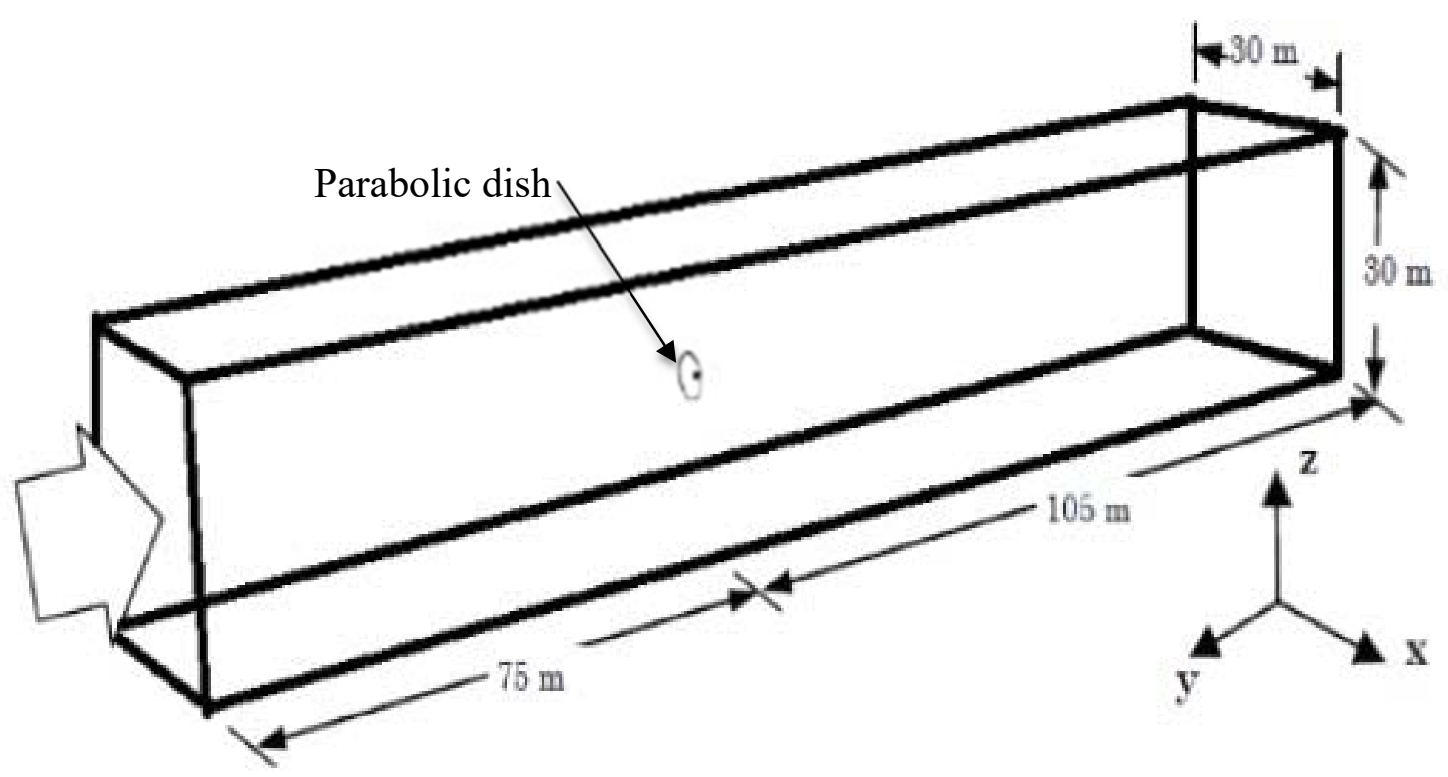

Fig 2: Computational domain

The wind flow over the system was modelled using the commercial CFD program ANSYS CFX 15.0.7 and the Shear Stress Transport (SST) turbulence model. The SST model has been shown to be one of the most accurate two-equation models for separation prediction and has been successfully used for studies of wind flow over parabolic troughs (ANSYS, 2013; Paetzold et al., 2015). In determining the heat loss it was assumed the system was subject to a free stream wind velocity of $5 \mathrm{~m} / \mathrm{s}$ and an ambient temperature of $25^{\circ} \mathrm{C}$ (a Reynolds number of $\sim 1.9 \times 10^{6}$ using the dish diameter as the characteristic length). Further, the internal cavity walls of the receiver were modelled as isothermal walls with a temperature of $600^{\circ} \mathrm{C}$ with buoyancy effects included in the calculation, to account for natural convection. Finally, steady state simulations were performed by changing the angle of the dish relative to the wind, from $90^{\circ}$ (direct impingement of the wind on the mirrored surface) to $0^{\circ}$ (dish aperture facing directly upwards) to $-90^{\circ}$ (wind impinging on the back surface of the mirrors).

\subsection{Experimental Setup}

To observe the behavior of turbulent flow around the dish a series of wind tunnel experiments were performed using a scaled down version of the large dish, with a model diameter of $150 \mathrm{~mm}$ $\left(\operatorname{Re} \sim 4 \times 10^{4}\right)$. In order to get a smooth finish a nylon model of the dish was manufactured using a three dimensional printing system. The model was mounted on a stand in the test section by means of $180 \mathrm{~mm}$ long steel rod that allowed for rotation around the x-axis (Fig 2). The test section of the wind tunnel was $500 \mathrm{~mm} \times 500 \mathrm{~mm}$ in cross section, and extended $500 \mathrm{~mm}$ upstream and $1000 \mathrm{~mm}$ downstream of the model. 
After testing of the wind tunnel to ensure homogenous flow, a series of smoke visualization experiments were performed to qualitatively validate the flow fields observed in the simulations. In order to diffuse the smoke in parallel lines inside the wind tunnel, a smoke rake was used and images of the smoke flow were captured in the presence of a green laser light sheet by a digital SLR camera. A schematic representation of the experimental setup is shown in Fig 3 .

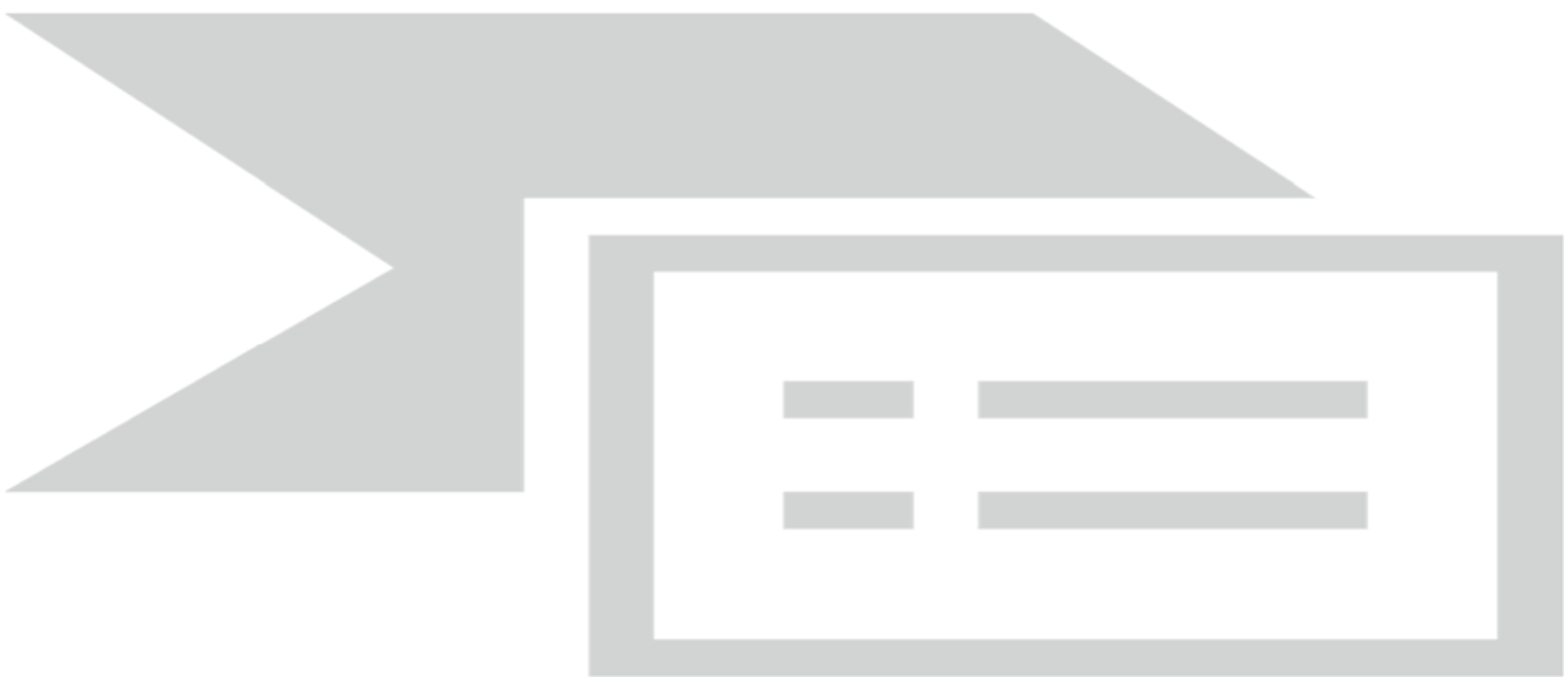

\section{Results and discussion}

Fig 3: Schematic drawing of the experimental bench

\subsection{Examination of flow features}

As mentioned previously, CFD simulations were performed for the parabolic dish system at different pitch angles while visualization of the turbulent flow around a parabolic dish structure was performed using smoke thus allowing the simulation results to be verified qualitatively. For different tilt angles of the dish, velocity streamlines along the center plane of the CFD simulation domain (with flow moving from left to right) were compared with the smoke streak lines as shown in Figs 4-12.

The flow around the dish shows markedly different flow structures with different tilt angles. Starting from the case when flow is perpendicular to the aperture plane of the dish, i.e. $90^{\circ}$ tilt angle; an accumulation of smoke in front panel of the dish can be seen (Fig. 4) due to the dish structure blocking the horizontal movement of air. Under these conditions, the local velocity becomes normal to the original flow near the dish's edge. In turn the velocity in the front of dish structure moves towards a stagnation condition and at the edges of dish structure the velocity values increase to maintain continuity. As a result, the velocity behind the dish is reduced and two strong recirculating vortices are generated due to reduced pressure in the dish's wake.

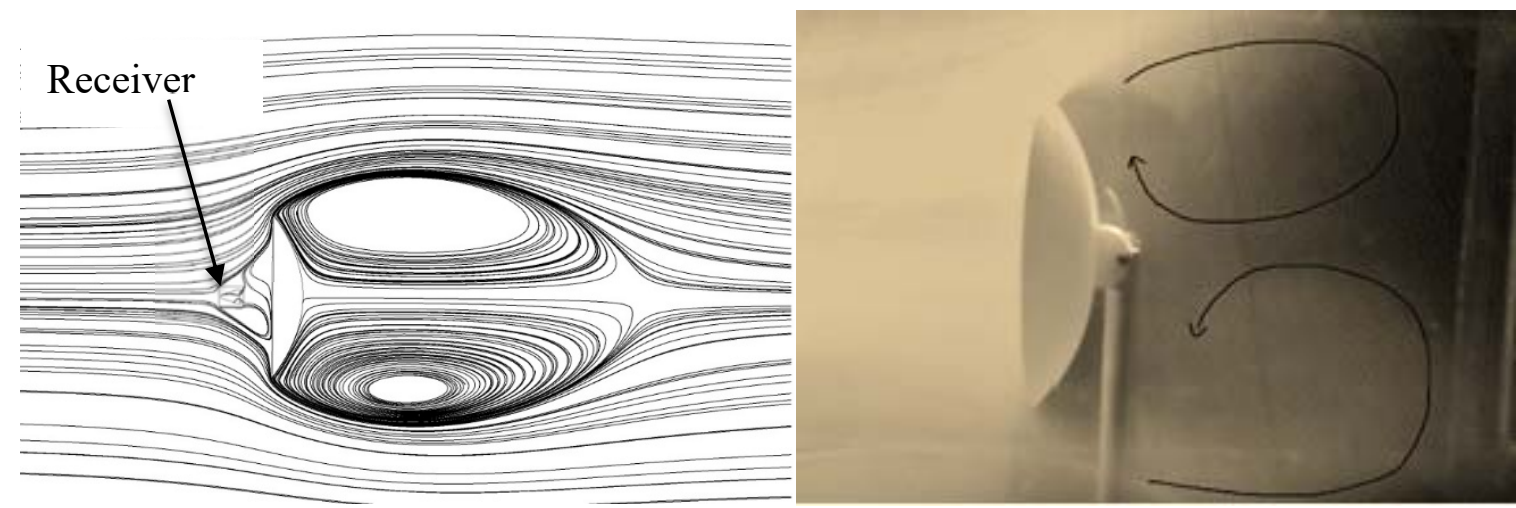

Fig 4: CFD streamlines and flow visualization for $90^{\circ}$ tilt angle 
By changing the tilt angle to $60^{\circ}$, the negative pressure creates flow separation at the upper and lower edge of dish, resulting in two large vortices behind the dish. From the streamlines shown in Fig 5, it can be seen that as the velocity increases at the upper and lower edge this leads towards a flow separation. Similarly, Fig 6 shows flow separation occurring from the upper edges, creating a vortex behind the dish, at a pitch angle of $45^{\circ}$.

However as the angle is reduced further, to a tilt angle of $30^{\circ}$ (Fig 7), the flow becomes more uniform and there is no major flow separation except a smaller flow recirculation region near to the lower portion of dish. As a result, the flow orientation is mainly upward due to the low pressure generated by the acceleration of the flow over the dish. The recirculation regions have effectively vanished as the dish reaches a $0^{\circ}$ tilt angle (Fig 8), and it suggests that the dish does not affect the air flow on the top side of dish for this orientation. Under these conditions, the receiver location would be in such a position that the shape of the dish would not influence the flow near it.
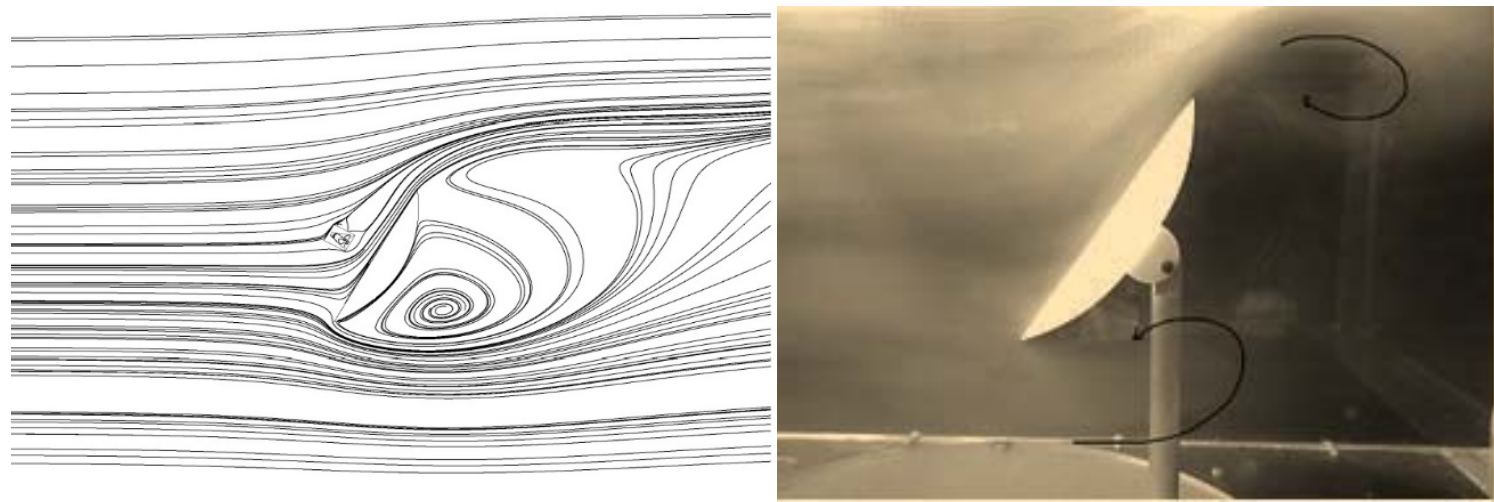

Fig 5: CFD streamlines and flow visualization for $60^{\circ}$ tilt angle

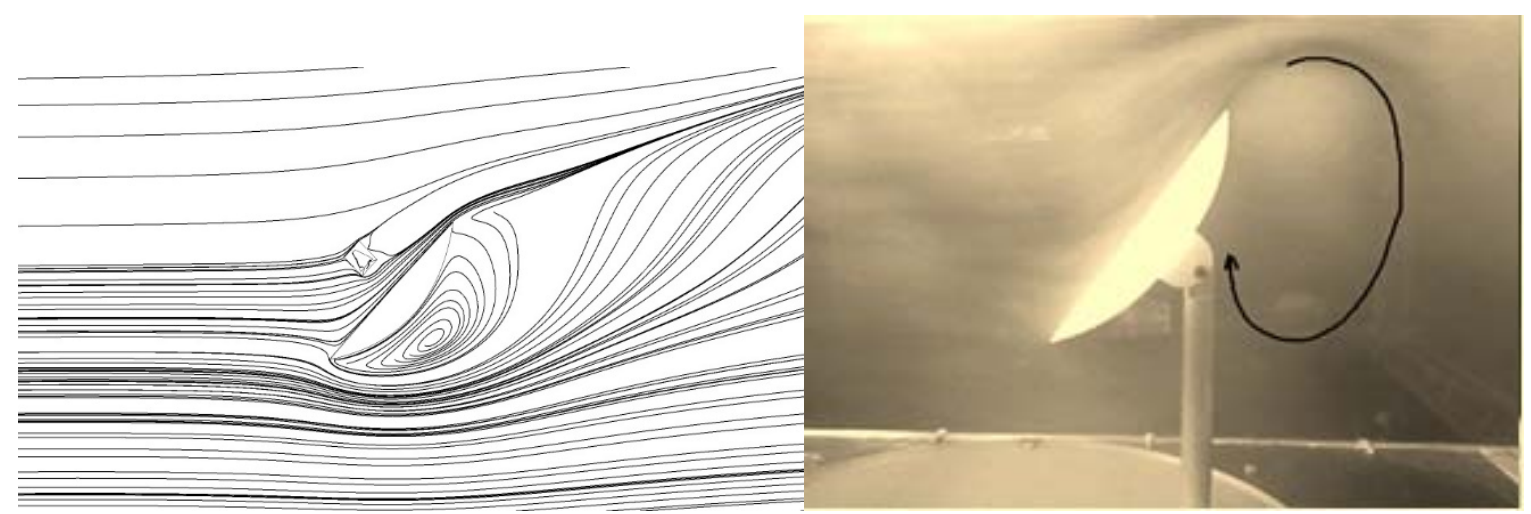

Fig 6: CFD streamlines and flow visualization for $45^{\circ}$ tilt angle

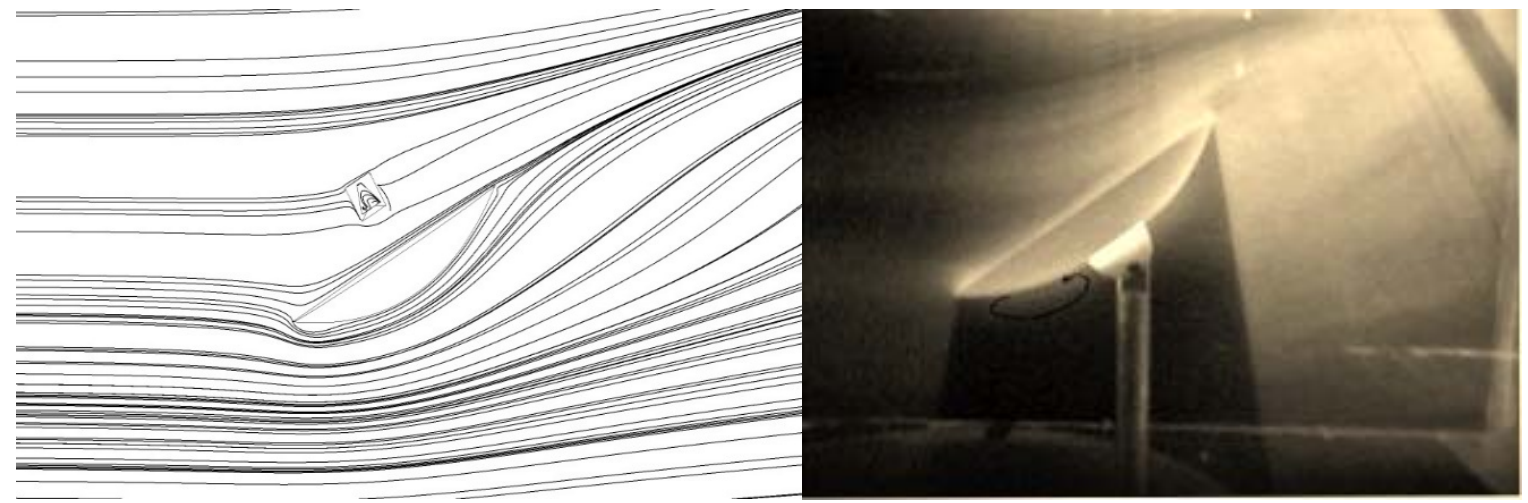

Fig 7: CFD streamlines and flow visualization for $30^{\circ}$ tilt angle 

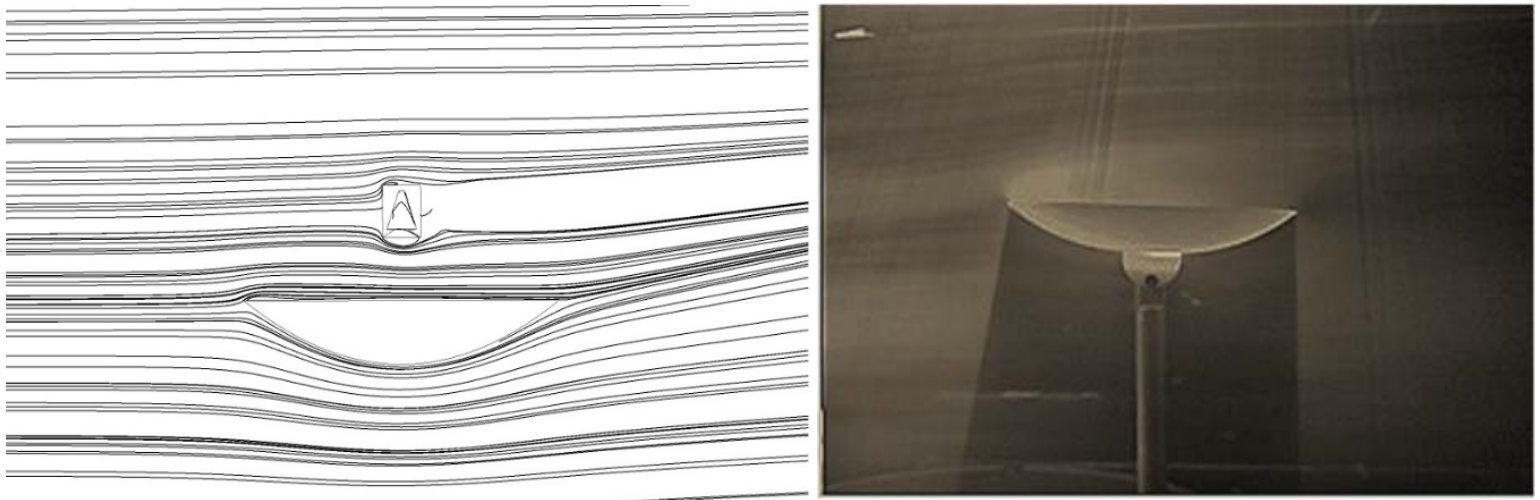

Fig 8: CFD streamlines and flow visualization for $0^{\circ}$ tilt angle

Now, by changing the tilt angle to $-30^{\circ}$, such that the flow is from the backside of the dish structure, the dish creates a strong vortex in its wake. As is visible in the Fig 9, smoke is trapped behind the dish and rotates locally. This smoke cloud is especially visible when the smoke generation is stopped, and takes a significant amount of time for the cloud to disperse. Thus, the local velocity is slow, and it is important to note that this is where the cavity receiver would be. This would suggest that for this orientation natural convection would be significant whereas for other orientations the forced component might be dominant.

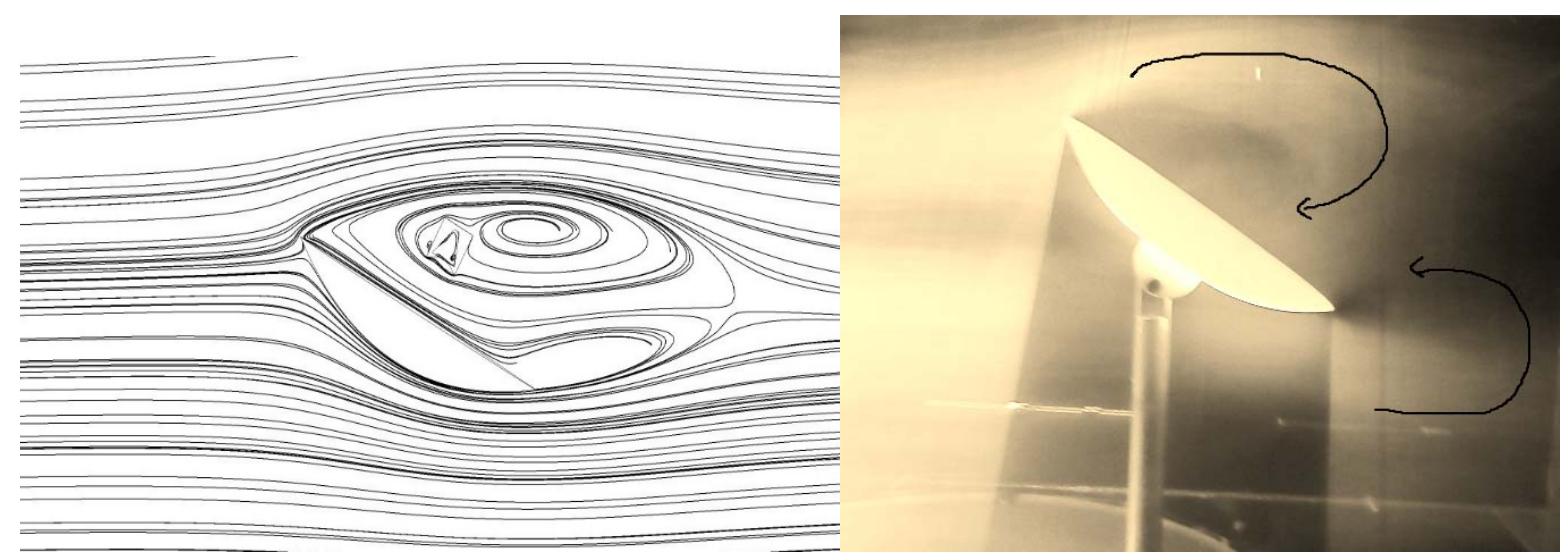

Fig 9: CFD streamlines and flow visualization for $-30^{\circ}$ tilt angle

By increasing the tilt angle to $-45^{\circ}$, again a large recirculation can be viewed in the wake region of dish creating a disorderly airflow in this area, while a small recirculation can be viewed near the lower edge (Fig. 10). Similar to $-30^{\circ}$ tilt angle, the local flow velocity behind the dish structure reduces sharply. In this respect, it is apparent that the flow near the aperture is dominated by the tangential components for tilt angles in the range $45^{\circ}$ to $-45^{\circ}$. Fig 11 shows the flow pattern for a tilt angle of $-60^{\circ}$, showing the high velocities at the edges of dish structure producing the negative pressure in the vicinity of the wake region. The negative pressure generates strong vortices behind the dish and like a $90^{\circ}$ tilt angle, the circulation region increases sharply at a tilt angle of $-90^{\circ}$ generating two large vortices behind the dish structure (Fig. 12). 


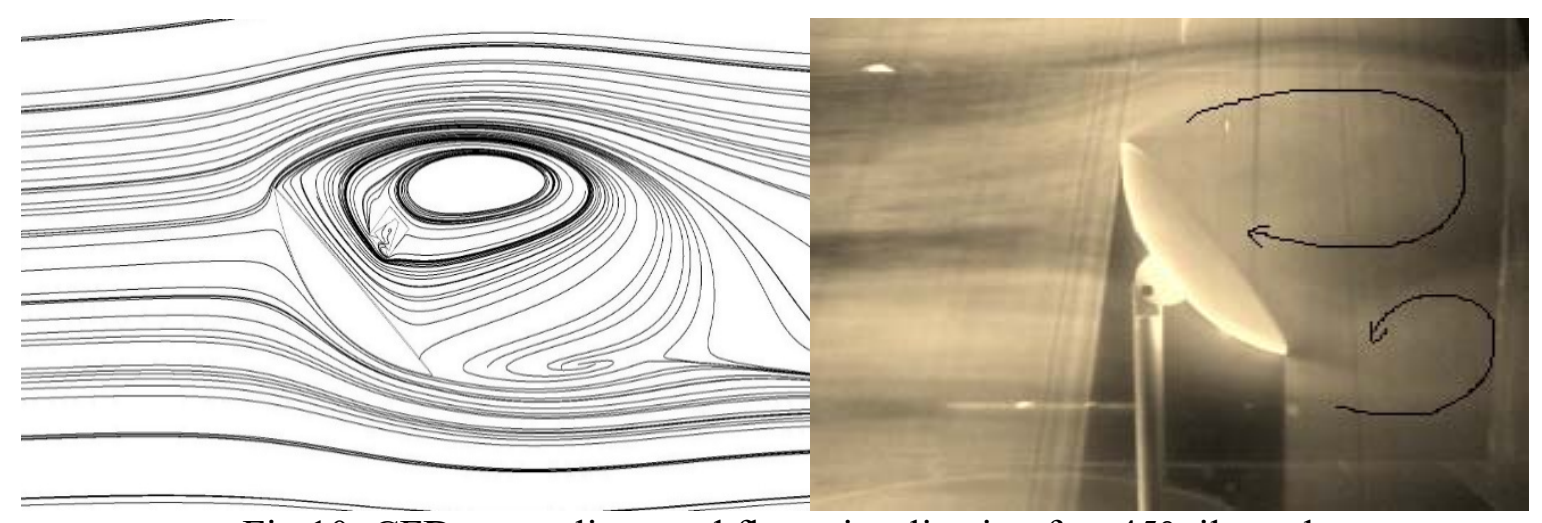

Fig 10: CFD streamlines and flow visualization for $-45^{\circ}$ tilt angle

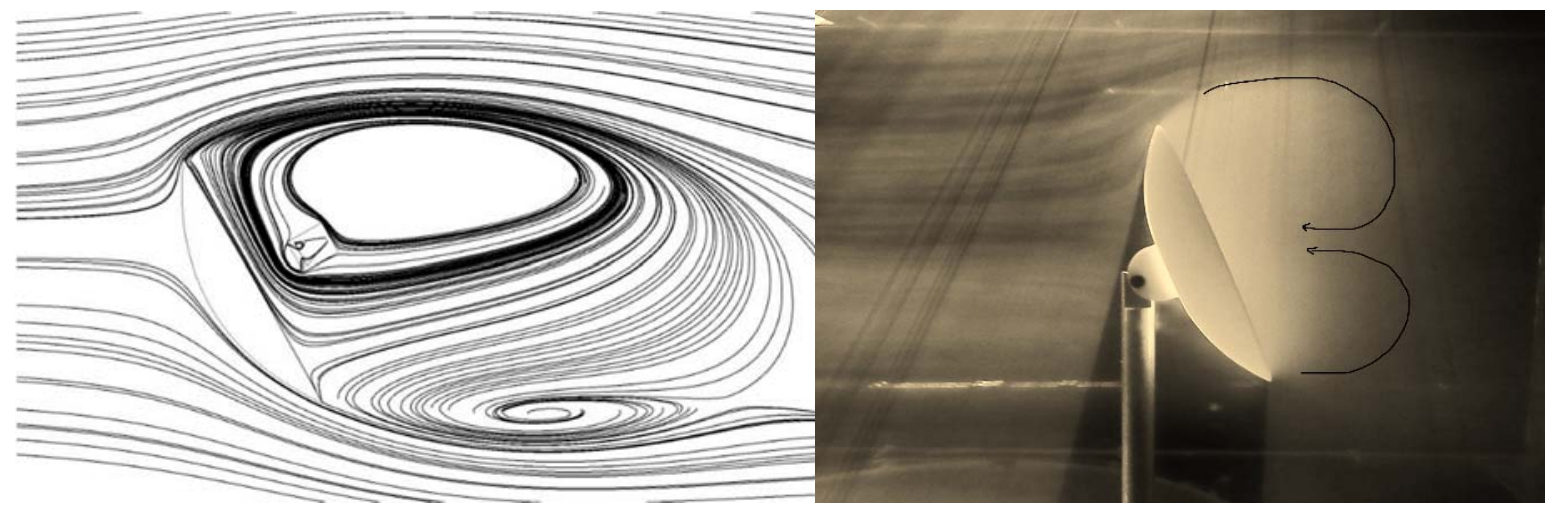

Fig 11: CFD streamlines and flow visualization for $-60^{\circ}$ tilt angle

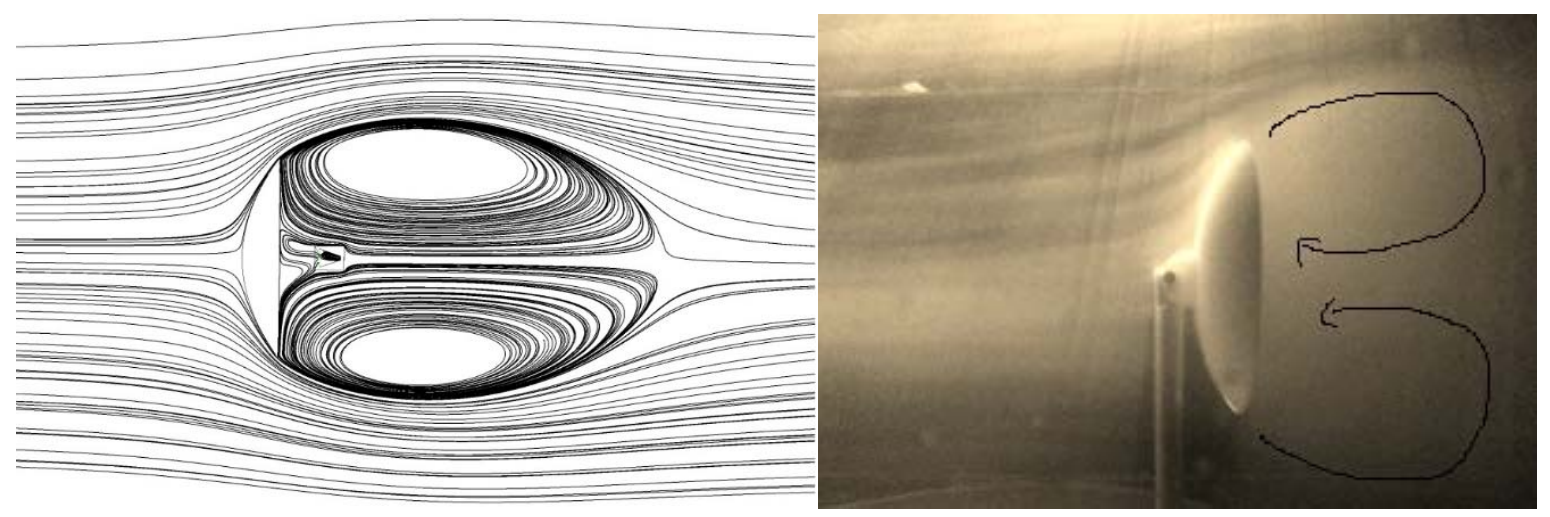

Fig 12: CFD streamlines and flow visualization for $-90^{\circ}$ tilt angle

In examining these images, the streak lines illustrate a shear layer that is also present in the simulation results. From these it can be seen that the shear layer's trajectory is dependent on the tilt angle of dish structure. In turn, the shear layer disturbance at the edge of the dish structure is indicative of the drag force acting on the dish. Based on these qualitative observations of the CFD streamlines and flow visualization, it was decided to examine the drag and lift coefficients determined from the simulations as a quantitative comparison.

The results shown in Figures 13a and $b$ compare the predicted drag and lift values from this simulation with the experimental values obtained by Wagner (1996) and the analytical expression proposed by Christo (2012). For this study, the coefficients were found to correspond well with the drag and lift coefficients of these previous studies, suggesting that the CFD is also quantitatively matching the flow effects of the parabolic dish in addition to the qualitative observations presented previously. 


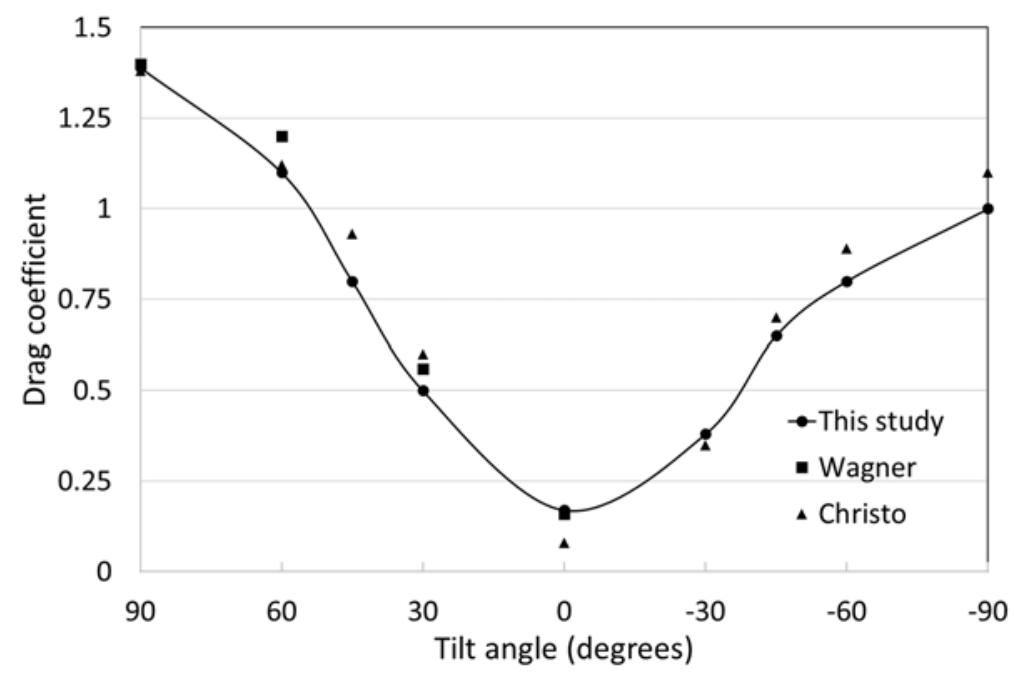

Fig 13a: Comparison of drag coefficient at different tilt angles

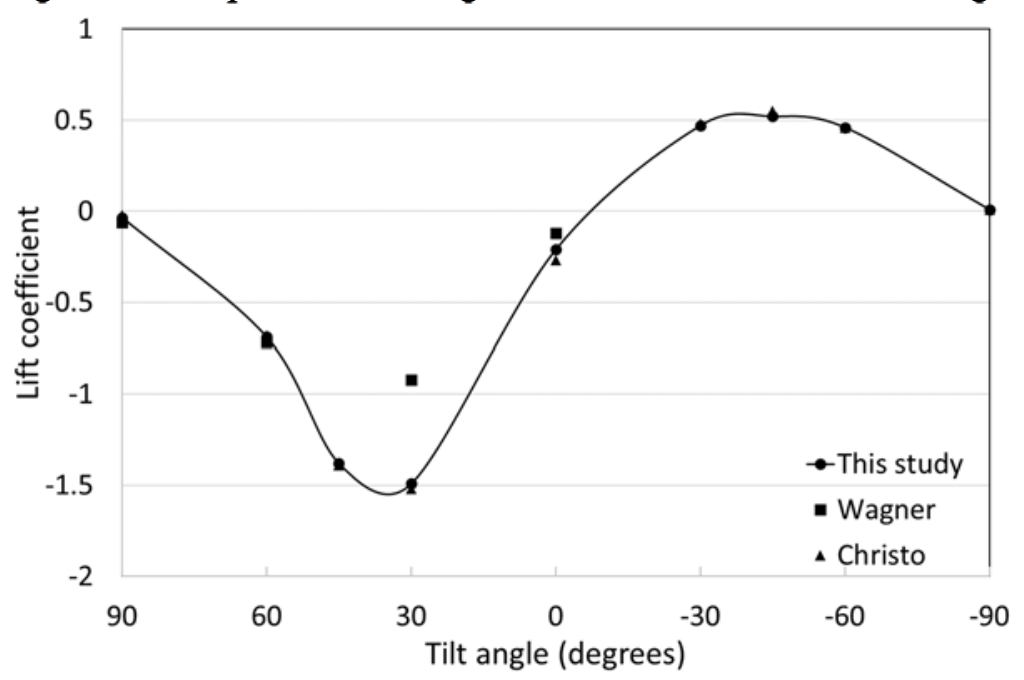

Fig 13b: Comparison of lift coefficient at different tilt angles

\subsection{Investigation of heat loss from a combined parabolic dish cavity receiver}

Having validated the simulation scheme, the methodology was applied to the determination of the heat loss from the cavity receiver. Figure 14 compares the convective heat loss from the cavity (at a wall temperature of $600^{\circ} \mathrm{C}$ ) with the reults of Paitoonsurikarn and Lovegrove's (2006) study (neglecting the presence of the dish); simulation results from this study without the dish present, and finally, with the flow around the dish being accounted for. It can be seen that, for cases where the dish is neglected, the convective heat loss decreases as the tilt angle moves from $+/-90^{\circ}$ (cavity axis aligned horizontally) to $0^{\circ}$ (cavity facing downward), which is to be expected as at $0^{\circ}$ the bouyancy forces are at their maximum.

However, what is most noticeable is that with the dish structure being present the convective heat loss is significantly lower (up to 40\%) than the cases where it is absent, except for the case of a zero degree tilt angle. At a zero degree tilt angle, the dish structure does not markedly affect the air flow on the top side of dish (as shown in Fig. 8) and as a result there is no impact on the flow near the cavity receiver or the heat loss from the cavity. 


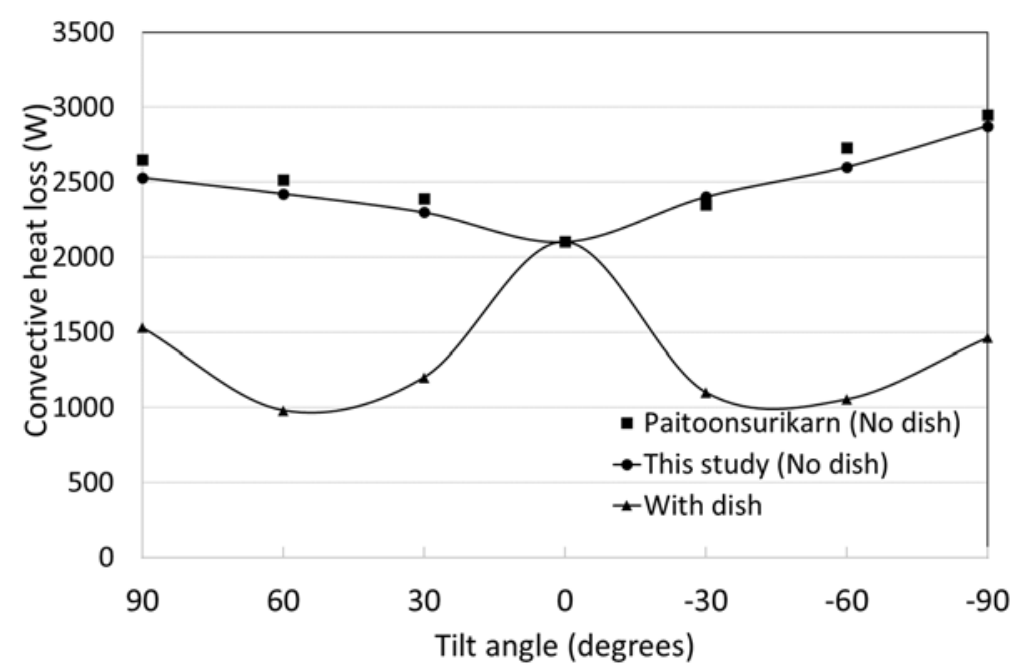

Fig 14: Heat loss from the parabolic dish receiver

Exploring this further, the observations of the flow structure due to the dish showed that there was a significant disturbance to the flow field, as such the convection heat losses from the cavity receiver might be forced or mixed convection subject to the tilt angle of the dish. In most of the cases examined the global Richardson number was less than unity ( $\mathrm{Ri}<<1)$, implying that forced convection was significant. However, for tilt angles of $-45^{\circ}$ to $-90^{\circ}$, the cavity receiver is surrounded by a large recirculation in the wake region of parabolic dish (as illustrated in Figs 10-12) and hence their is a marked difference in the heat loss. This difference arises due to the fact that without the dish structure being present the cavity is exposed to strong forced convective flows, whereas in the wake the heat loss is driven by the weaker natural convection mechanism. Similarly, for the dish facing into the wind, a region of low velocity air is present due to the bulk flow having to move around the dish meaning that a similar outcome is observed.

However, what is interesting to note is that for tilt angles between $+/-30^{\circ}$ and $0^{\circ}$ the heat loss increases. This can be attributed to the receiver moving into the free stream and being subject to stronger forced flow, rather than residing in the wake. That said, the overarching conclusion is that parabolic dish CSP systems designed without consideration to effect of the dish on heat loss are likely to be conservative and hence more expensive than they could be.

\section{Conclusion}

This study has shown a good agreement between quantitative and qualitative assessments of the flow field around a parabolic dish. The results indicate a significant disturbance to the local air velocity near the cavity receiver at all tilt angles, except in the case of parallel flow to the aperture of dish.

Furthermore, the results reveal a significant reduction in the convective heat loss from a cavity receiver in the presence of dish structure subject to a forced air flow. In particular, the convective heat loss shows a dependence on the tilt angle, with a minimum convective loss when the cavity receiver is facing straight downward. Based on results, it can be concluded that the orientation of a parabolic dish has a significant effect on the heat loss from cavity receivers.

Finally, the results demonstrate the need to consider the influence of the flow around the dish when determining the heat loss from a parabolic dish receiver to avoid designing overly conservative and hence costly CSP systems. 


\section{References}

ANSYS Inc. 2013. CFX User guide, ANSYS, Canonsburg.

Christo, F., 2012. Numerical modelling of wind and dust pattern around a full-scale paraboloidal solar dish. Renewable Energy 32 (1), 356-366.

Clausing, A. M., 1981. An Analysis of Convective Losses from Cavity Solar Central Receiver. Solar Energy 27 (4), 295-300.

Clausing, A. M., Waldvogel, J.M., Lister, L.D., 1987. Natural convection from isothermal cubical cavities with a variety of side facing apertures. ASME Journal of Heat Transfer, 109 (2), 407-412.

Le Quere, P., Humphery, J. A., Sherman, F. S., 1981a. Numerical calculation of thermally driven two-dimensional unsteady laminar flow in cavities of rectangular cross section. Numerical Heat Transfer Part B, 4 (3), 249-283.

Le Quere, P., Penot, F., Mirenayat, M., 1981b. Experimental study of heat loss through natural convection from an isothermal cubic open cavity. Sandia National Laboritories Report. SAND81-8014. USA.

Leibfried, U., Ortjohann, J., 1995. Convective Heat Loss from Upward and Downward-Facing Cavity Solar Receivers: Measurements and Calculations. Journal of Solar Energy Engineering 117 (2), 75-84.

Lupfert, E., Geyer, M., Schiel, W., Esteban, A., Osuna, R., Zarza, E., 2001, EUROTHROUGH design issues and prototype testing at PSA, Proceedings of Solar Forum 2001: Solar Energy: The Power to Choose, Washington DC.

Ma, R. Y., 1993. Wind effects on convective heat loss from a cavity receiver for a parabolic concentrating solar collector. Sandia National Laboratories Report. SAND92-7293. USA.

Paetzold, J., Cochard, C., Fletcher, D., Vassallo, A. 2015., Wind Engineering Analysis of Parabolic Trough Collectors to Optimise Wind Loads and Heat Loss, Energy Procedia, 69, $168-177$

Paitoonsurikarn, S., Lovegrove, K., 2003. On the study of convection loss from open cavity receiver in solar paraboloidal dish applications. Proceeding of 41st Conference of the Australia and New Zealand Solar Energy Society (ANZSES). Melbourne, Australia.

Paitoonsurikarn, S., Taumoefolau, T., Lovegrove, K., 2004. Estimation of convection heat loss from paraboloidal dish cavity receivers. Conference of Australia and New Zealand Solar Energy Society (ANZSES). Perth, Australia.

Paitoonsurikarn, S., Lovegrove, K., 2006. Effect of paraboloidal dish structure on the wind near a cavity receiver. Proceedings of the 44th Annual Conference of the Australian and New Zealand Solar Energy Society. Canberra, Australia.

Siebers, D. L., Kraabel, J. S.,1984. Estimating convective energy losses from solar central receivers. Sandia National Laboratories Report. SAND 84-8717. 
Steinfield, A., 2005. Solar thermochemical production of hydrogen-a review. Solar Energy 78 (5), 603-615.

Stine, W. B., McDonald, C. G., 1989. Cavity Receiver Heat Loss Measurements, Proceedings of the International Solar Energy Society Solar World Congress, vol. 2, 1318-1322, Kobe, Japan, 1989

Tyner, C.E., Kolb, G.J., Geyer, M., Romero, M., 2001. Concentrating solar power in 2001- an IEA/Solar PACES summary of present status and future prospectus. SolarPACES, www.solarpaces.org (Last cited 05/09/2015).

Wagner, G.L., 1996. Solar Concentrator Wind Loadings. MSc Thesis, Texas Tech University.

Wu, S.Y., Xiao, L., Cao, Y. Li, Y-R, 2010, Convection heat loss from cavity receiver in parabolic dish solar thermal power system: A review, Solar Energy 84 (8), 1342-1355 